\title{
On the Impact of Atmospheric Correction on Lossy Compression of Multispectral and Hyperspectral Imagery
}

\author{
Qian Du, Senior Member, IEEE, James E. Fowler, Senior Member, IEEE, and Wei Zhu, Student Member, IEEE
}

\begin{abstract}
Reflectance data is often preferred to radiance data in applications of multispectral and hyperspectral imagery in which subtle spectral features are analyzed. In such applications, atmospheric correction, the process which provides radianceto-reflectance conversion, plays a prominent role in the datadistribution and archiving pipeline. Lossy compression, often in the form of the JPEG2000 standard, will also likely factor into the distribution and archiving data flow. The relative position of data compression with respect to atmospheric correction is considered and evaluated with experimental results on both multispectral and hyperspectral imagery, and recommendations on an appropriate order for compression in the data-flow chain are made.
\end{abstract}

Index Terms - data compression, atmospheric correction, multispectral imagery, hyperspectral imagery

\section{INTRODUCTION}

The vast quantities of data typical to multispectral and hyperspectral imagery presents great challenges in transmission, storage, and analysis. Consequently, data compression is becoming a common practice during the archiving and distribution of such imagery. In general, compression can be lossy or lossless. When the application in which a specific dataset might be used in the future is unknown, it may be appropriate to apply a lossless or near-lossless compression technique (e.g., [1-3]). On the other hand, when the application is known, lossy compression may provide a much higher amount of compression while preserving the information in the original data that is essential to the application. For lossy compression of hyperspectral imagery, there is currently great interest surrounding the JPEG2000 standard [4, 5] which has been demonstrated to yield exceedingly efficient compression performance (e.g., [6-8]).

Whether radiance or reflectance data is preferred will depend on the specific application intended for the data. For instance, for detection or classification, radiance data can be used without significantly influencing the overall detection or classification accuracy, particularly when the size of the treated area is not very large [9]. On the other hand, for applications in which subtle spectral features are very important-such as the retrieval of crop chlorophyll content or leaf-area indexreflectance data is often used $[10,11]$. Atmospheric correction, the process that provides radiance-to-reflectance conversion, thus plays a prominent role in the data-distribution and archiving process in these latter applications. If lossy compression

The authors are with the Department of Electrical \& Computer Engineering and the GeoResources Institute, Mississippi State University, Starkville, MS. is also to be applied, there are two possible locations for it in the data-flow pipeline-either before or after atmospheric correction. In other words, when the ultimate application of the data necessitates atmospheric correction, the choice must be made between compressing either radiance data or reflectance data. The question then arises as to which option is better.

In this correspondence, we conduct a series of studies to provide some insight on this issue surrounding lossy compression with respect to atmospheric correction. Below, we first briefly overview JPEG2000 which has become the preferred paradigm for lossy compression of multispectral and hyperspectral imagery. We then consider the issue of atmospheric correction and present experimental results examining its impact on lossy compression.

\section{JPEG2000 COMPRESSION}

For coding multispectral or hyperspectral imagery, a 3D discrete wavelet transform (DWT) is most often applied to the data cube in the form of a wavelet-packet transform-a 1D DWT applied spectrally followed by a 2D spatial DWT. It is known that this particular wavelet-packet decomposition is near-optimal for hyperspectral imagery and outperforms, in particular, the dyadic decomposition consisting of recursive decomposition of the lowpass subband cube $[6,12]$. Alternatively, principal component analysis (PCA) has also been widely used for spectral decorrelation (e.g., [7, 8]). Consequently, when we use JPEG2000 for multispectral and hyperspectral compression, we have two variants according to the specific spectral transform applied. In particular, we use "DWT+JPEG2000" to denote JPEG2000 with a 1D DWT applied spectrally followed by a 2D DWT spatially. If, instead, PCA is employed for spectral decorrelation (with the 2D DWT still applied spatially), we denote this as "PCA+JPEG2000."

For PCA+JPEG2000, we generate the PCA transform matrix and data mean vector in MATLAB; the JPEG2000 encoder then performs the spectral transform and embeds the mean vector as well as the inverse-transform matrix into the JPEG2000 bitstream in the form of a multicomponent transform (MCT) marker as specified in Part 2 of the JPEG2000 standard [5]. The encoder automatically allocates rate simultaneously across the resulting principal components; i.e., post-compression rate-distortion (PCRD) optimization [13] is applied simultaneously to all codeblocks to optimally truncate the embedded bitstream for each codeblock. In the reconstruction process, the JPEG2000 decoder automatically extracts 
the transform matrix and mean vector and then applies them appropriately after the bitstream has been decoded.

Previously [8], we have found that PCA can significantly outperform the DWT for spectral decorrelation of radiance data. For example, Fig. 1 indicates up to a 5-dB gain in performance for PCA+JPEG2000 as compared to DWT+JPEG2000 for a hyperspectral radiance dataset. This performance increase is due to the fact that PCA typically obtains a higher degree of decorrelation than does the DWT coupled with the fact that JPEG2000 does not exploit any residual spectral correlation after the spectral transform is applied. Below, we investigate whether this advantage still holds for PCA when atmospheric correction is considered and distortion is measured with respect to reflectance data.

\section{RAdiAnCE Versus ReFlectance DatA}

To investigate how atmospheric correction influences datacompression performance, two cases are compared as illustrated in Fig. 2. In Case 1 (Fig. 2(b)), radiance data is converted to reflectance data, the reflectance data is compressed, and the resulting compressed bitstream is decompressed to produce a reconstructed reflectance dataset. As an alternative, in Case 2 (Fig. 2(c)), radiance data is compressed and then decompressed with the reconstructed radiance being corrected to produce reflectance. In both cases, the reconstructed reflectance dataset that is the end result of the processing is compared to true reflectance data, i.e., reflectance as obtained by applying atmospheric correction to the radiance without any intervening compression (Fig. 2(a)).

There are several software packages available for atmospheric correction, including Atmospheric REMoval program (ATREM), Atmospheric CORrection Now (ACORN), and the Fast Line-of-sight Atmospheric Analysis of Spectral Hypercubes (FLAASH) [14, 15]. In the work here, we use FLAASH, which is an add-on module for ENVI, a widely used software package for remote-sensing data processing and analysis. FLAASH is based on MODTRAN-4, a software package developed by the Air Force Phillips Lab and Spectral Sciences, Inc. (SSI). It operates in the 0.4-2.5 $\mu \mathrm{m}$ spectral range, so it can be used for all the currently available optical multispectral and hyperspectral sensors. FLAASH is capable of providing accurate, physics-based derivation of apparent atsurface reflectance from at-sensor radiance through derivation of atmospheric properties such as surface albedo, surface altitude, water-vapor column, aerosol, cloud optical depths, and surface and atmospheric temperatures.

\section{EXPERIMENTAL RESULTS}

We use two datasets to gauge performance for both multispectral and hyperspectral imagery. For a multispectral dataset, we use Landsat 7 data of the Jasper Ridge scene taken in 1999 with spatial size of $512 \times 512$ and 6 optical bands. For a hyperspectral dataset, we use AVIRIS data of the Jasper Ridge scene taken in 1998 of size $512 \times 614$ with 224 optical bands. ${ }^{1}$ For the JPEG2000 coder, we use Kakadu ${ }^{2}$ Version 5.11

\footnotetext{
${ }^{1}$ Both Jasper Ridge datasets are available on the ENVI FLAASH tutorial disk.

${ }^{2}$ http: //www.kakadusoftware.com
}

which implements Parts 1 and 2 of the JPEG2000 standard. All DWTs use the popular 9/7 biorthogonal DWT.

For the multispectral dataset, Tables I and II tabulate the $\mathrm{SNR}^{3}$ and spectral angles ${ }^{4}$ (both mean and standard deviation (std) when compressing reflectance (Case 1) and radiance (Case 2) data. We can see that SNR values are slightly higher and spectral angles slightly smaller for Case 1 . These results suggest that one should conduct atmospheric correction before lossy compression if both processes are needed for a multispectral image. The results also indicate that PCA+JPEG2000 outperforms DWT+JPEG2000 in all cases. For the hyperspectral datasets, Tables III and IV tabulate the SNR and spectral angles for the two cases. Again, the performances for Case 1 are superior to those for Case 2; the difference is, in fact, much more pronounced for the hyperspectral data than it is for the multispectral data. PCA+JPEG2000 also outperforms DWT+JPEG2000 in all cases for the hyperspectral data as well.

\section{Conclusions}

For situations in which atmospheric correction is a necessary step prior to data analysis, it is recommended to conduct atmospheric correction before lossy data compression; i.e., compressing reflectance data is more appropriate than compressing radiance data. In essence, lossy compression algorithms, such as the JPEG2000 encoder used here, are designed to minimize the error (typically measured in a meansquared sense) between the original data and its reconstruction; consequently, lossy compression should be applied in the domain in which the error is most relevant. That is to say, if processing is to take place in the "reflectance domain" (i.e., after atmospheric correction), then compression should take place in that domain as well. If, on the other hand, compression is applied in the radiance domain, it is unlikely that minimal radiance-domain error will translate into minimal reflectance-domain error due to the complex, nonlinear nature of the atmospheric-correction process (note that if atmospheric correction were a linear operation, then equivalent results for both reflectance- and radiance-domain compression would occur for squared-error-based measures like the SNR due to Parseval's theorem). This issue is particularly important in the case of hyperspectral data since the larger number of bands apparently creates a greater degree of nonlinearity in the atmospheric-correction process.

\section{REFERENCES}

[1] F. W. Chen, "Archiving and distribution of 2-D geophysical data using image formats with lossless compression," IEEE Geoscience and Remote Sensing Letters, vol. 2, no. 1, pp. 64-68, January 2005.

[2] S.-E. Qian, M. Bergeron, I. Cunningham, L. Gagnon, and A. Hollinger, "Near lossless data compression onboard a hyperspectral satellite," IEEE Transactions on Aerospace and Electronic Systems, vol. 42, no. 3, pp. 851-866, July 2006.

[3] H. Wang, S. D. Babacan, and K. Sayood, "Lossless hyperspectralimage compression using context-based conditional average," IEEE Transactions on Geoscience and Remote Sensing, vol. 45, no. 12, pp. 4187-4193, December 2007.

\footnotetext{
${ }^{3}$ Throughout all our results, we define SNR as the log ratio between the variance of the original image and that of the difference image.

${ }^{4}$ Spectral angle denotes the angle between two hyperspectral pixel vectors.
} 


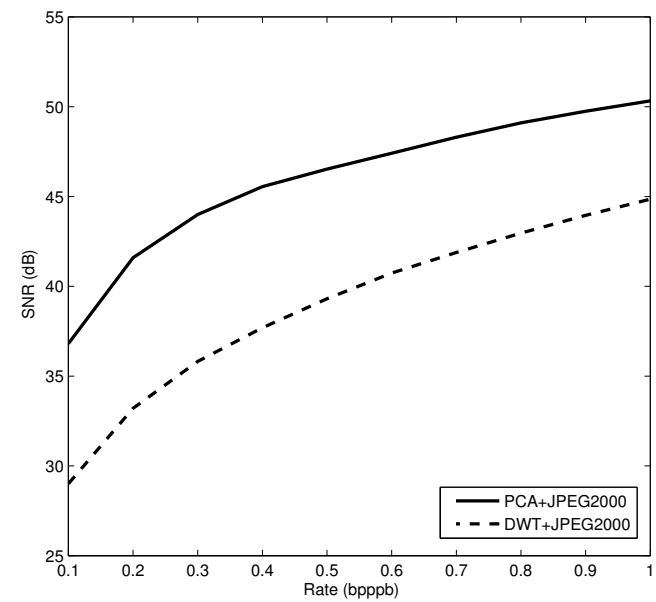

Fig. 1. Rate-distortion performance of JPEG2000 compression of the AVIRIS Jasper Ridge radiance dataset of size $512 \times 512$ and 224 bands (from [8]) Distortion is measured as signal-to-noise ratio (SNR) and rate in terms of bits per pixel per band (bpppb).

[4] Information Technology-JPEG 2000 Image Coding System-Part 1: Core Coding System, ISO/IEC 15444-1, 2000.

[5] Information Technology-JPEG 2000 Image Coding System-Part 2. Extensions, ISO/IEC 15444-2, 2004

[6] B. Penna, T. Tillo, E. Magli, and G. Olmo, "Transform coding techniques for lossy hyperspectral data compression," IEEE Transactions on Geoscience and Remote Sensing, vol. 45, no. 5, pp. 1408-1421, May 2007.

[7] — "Hyperspectral image compression employing a model of anomalous pixels," IEEE Geoscience and Remote Sensing Letters, vol. 4, no. 4 pp. 664-668, October 2007.

[8] Q. Du and J. E. Fowler, "Hyperspectral image compression using JPEG2000 and principal component analysis," IEEE Geoscience and Remote Sensing Letters, vol. 4, no. 2, pp. 201-205, April 2007.

[9] D. A. Landgrebe, Signal Theory Methods in Multispectral Remote Sensing. Hoboken, NJ: Wiley-Interscience, 2003.

[10] S.-E. Qian, A. Hollinger, M. Bergeron, I. Cunningham, C. Nadeau, G. Jolly, and H. Zwick, "A multidisciplinary user acceptability study of hyperspectral data compressed using an on-board near lossless vector quantization algorithm," International Journal of Remote Sensing, vol. 26, no. 10, pp. 2163-2195, May 2005.

[11] B. Hu, S.-E. Qian, D. Haboudane, J. R. Miller, A. B. Hollinger, N. Tremblay, and E. Pattey, "Retrieval of crop chlorophyll content and leaf area index from decompressed hyperspectral data: The effects of data compression," Remote Sensing of Environment, vol. 92, no. 2, pp. 139-152, August 2004.

[12] J. E. Fowler and J. T. Rucker, "3D wavelet-based compression of hyperspectral imagery," in Hyperspectral Data Exploitation: Theory and Applications, C.-I. Chang, Ed. Hoboken, NJ: John Wiley \& Sons, Inc., 2007, ch. 14 , pp. $379-407$.

[13] D. Taubman, "High performance scalable image compression with EBCOT," IEEE Transactions on Image Processing, vol. 9, no. 7, pp. 1158-1170, July 2000.

[14] T. Cooley, G. P. Anderson, G. W. Felde, M. L. Hoke, A. J. Ratkowski, J. H. Chetwynd, J. A. Gardner, S. M. Adler-Golden, M. W. Matthew, A. Berk, L. S. Bernstein, P. K. Acharya, D. Miller, and P. Lewis, "FLAASH, a MODTRAN4-based atmospheric correction algorithm, its application and validation," in Proceedings of the International Geoscience and Remote Sensing Symposium, vol. 3, Toronto, Canada, June 2002, pp. 1414-1418.

[15] F. A. Kruse, "Comparison of ATREM, ACORN, and FLAASH atmospheric corrections using low-altitude AVIRIS data of Boulder, CO," in Proceedings of the $13^{\text {th }}$ JPL Airborne Geoscience Workshop, Pasadena, CA, March 2004

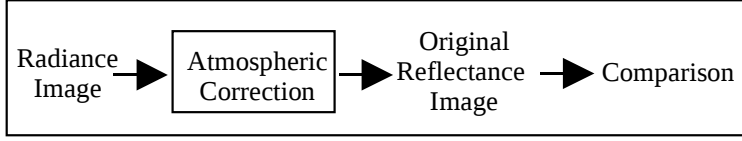

(a) True reflectance data for evaluating performance

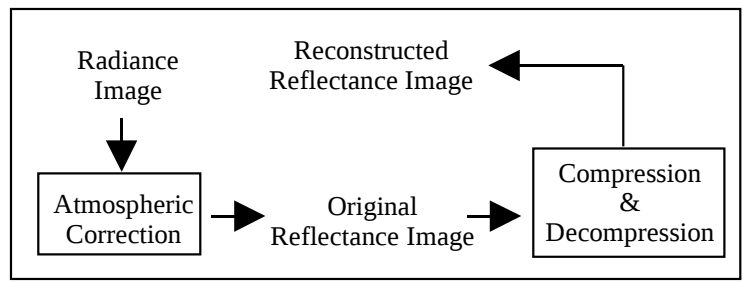

(b) Case 1: Reflectance data is compressed

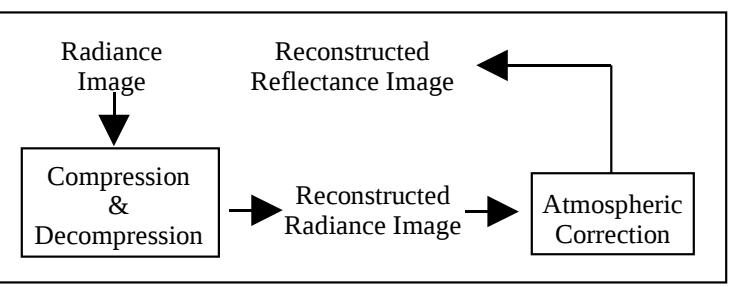

(c) Case 2: Radiance data is compressed

Fig. 2. Compression of reflectance versus compression of radiance.

TABLE I

SNR (DB) FOR MULTISPECTRAL IMAGERY

\begin{tabular}{|c|c|c|c|c|}
\hline \multicolumn{2}{|c|}{ Bitrate (bpppb) } & 0.1 & 0.5 & 1.0 \\
\hline \multirow{2}{*}{ PCA+JPEG2000 } & Case 1 & 13.49 & 19.68 & 24.29 \\
\cline { 2 - 5 } & Case 2 & 13.40 & 19.45 & 23.97 \\
\hline \multirow{2}{*}{ DWT+JPEG2000 } & Case 1 & 13.06 & 18.42 & 22.41 \\
\cline { 2 - 5 } & Case 2 & 13.03 & 18.34 & 22.27 \\
\hline
\end{tabular}

TABLE II

SPeCtral Angle (Degrees) FOR Multispectral IMAGery

\begin{tabular}{|c|c|c|c|c|c|}
\hline \multicolumn{3}{|l|}{ Bitrate (bppb) } & 0.1 & 0.5 & 1.0 \\
\hline \multirow{3}{*}{ PCA+JPEG2000 } & \multirow{2}{*}{ Case 1 } & mean & 5.35 & 3.15 & 2.06 \\
\cline { 2 - 6 } & & std & 6.03 & 3.72 & 2.53 \\
\cline { 2 - 6 } & \multirow{2}{*}{ Case 2 } & mean & 5.36 & 3.20 & 2.12 \\
\cline { 3 - 6 } & & std & 6.03 & 3.82 & 2.53 \\
\hline \multirow{3}{*}{ DWT+JPEG2000 } & \multirow{2}{*}{ Case 1 } & mean & 6.01 & 3.59 & 2.49 \\
\cline { 2 - 6 } & & std & 7.44 & 4.30 & 2.97 \\
\cline { 2 - 6 } & \multirow{2}{*}{ Case 2 } & mean & 5.92 & 3.65 & 2.50 \\
\cline { 3 - 6 } & & std & 7.29 & 4.43 & 3.09 \\
\hline
\end{tabular}

TABLE III

SNR (DB) FOR HYPERSPECTRAL IMAGERY

\begin{tabular}{|c|c|c|c|c|}
\hline \multicolumn{2}{|c|}{ Bitrate (bpppb) } & 0.1 & 0.5 & 1.0 \\
\hline \multirow{2}{*}{ PCA+JPEG2000 } & Case 1 & 25.74 & 34.74 & 40.05 \\
\cline { 2 - 5 } & Case 2 & 22.36 & 25.54 & 26.84 \\
\hline \multirow{2}{*}{ DWT+JPEG2000 } & Case 1 & 21.87 & 30.71 & 35.86 \\
\cline { 2 - 5 } & Case 2 & 9.35 & 16.57 & 21.65 \\
\hline
\end{tabular}

TABLE IV

SPECTRAL ANGLE (DEGREES) FOR HyPERSPECTRAL IMAGERY

\begin{tabular}{|c|c|c|c|c|c|}
\hline \multicolumn{3}{|c|}{ Bitrate (bpppb) } & 0.1 & 0.5 & 1.0 \\
\hline \multirow{3}{*}{ PCA+JPEG2000 } & \multirow{2}{*}{ Case 1 } & mean & 2.11 & 0.78 & 0.42 \\
\cline { 3 - 6 } & & std & 1.51 & 0.56 & 0.30 \\
\cline { 2 - 6 } & \multirow{2}{*}{ Case 2 } & mean & 2.85 & 2.16 & 1.88 \\
\cline { 3 - 6 } DWT+JPEG2000 & & std & 2.08 & 1.68 & 1.45 \\
\hline \multirow{3}{*}{ Case 1 } & mean & 3.15 & 1.22 & 0.68 \\
\cline { 3 - 6 } & & std & 2.06 & 0.81 & 0.47 \\
\cline { 3 - 6 } & \multirow{2}{*}{ Case 2 } & mean & 11.23 & 5.11 & 3.11 \\
\cline { 3 - 6 } & & std & 7.86 & 3.68 & 2.23 \\
\hline
\end{tabular}

